\title{
An efficient protocol for the purification and labeling of entire yeast septin rods from E.coli for quantitative in vitro experimentation
}

Christian Renz, Nils Johnsson and Thomas Gronemeyer ${ }^{*}$

\begin{abstract}
Background: The detailed understanding of the functions and mechanisms of the actin and microtubuli cytoskeleton depended, besides innovative methods in live cell imaging, on the purification and labeling of its constituents. This allowed researchers to quantitatively measure filament stability, the rates of filament turnover as well as the determination of the influence of cofactors on filament formation and structure. Septins form the least understood class of cytoskeletal structures in nearly all eukaryotic cells so far examined. In yeast, they comprise a family of proteins (Cdc3, Cdc10, Cdc11, Cdc12, Shs1) that form a co-polymeric, ring-like structure beneath the membrane. This ring serves as a template for the formation of a new bud neck and as a landing pat for proteins involved in polar growth and cytokinesis. Further progress in investigating the mechanisms of septin-structure formation and regulation is hampered by the lack of protocols to modify homogenous samples of purified septins with useful probes for in vitro biochemical studies.

Results: We present a protocol for the purification and labeling of yeast septin rods. The four individual septin subunits were co-expressed in E.coli. One subunit of the septin polymer was expressed as SNAP tag fusion protein allowing for rapid and stoichiometric labeling with derivatized Benzylguanine (BG). To demonstrate the applicability of our approach, we introduced two different SNAP tag substrates: septin rods labeled with fluorescent BG compounds enabled us to monitor the formation of filaments by fluorescence microscopy whereas BG-biotin was used to couple septin rods to a sensor chip for quantitative surface plasmon resonance binding experiments. In a first application, we determined the affinity and the binding kinetics of the yeast protein Bni5 to the individually coupled septin rods. In a further application we could demonstrate that a once formed septin rod hardly exchange its subunits.

Conclusions: The herein introduced protocol of purifying SNAP tag modified septins from E.coli allowed us to derivatize the obtained septin rods with probes for the further in vitro characterization of this class of cytoskeletal elements. The availability of a very diverse set of SNAP tag substrates should open the way to investigate different aspects of septin biochemistry in mechanistic detail.
\end{abstract}

Keywords: Septins, Protein purification, SNAP tag, Protein interactions

\footnotetext{
*Correspondence: thomas.gronemeyer@uni-ulm.de

Department of Biology, Institute of Molecular Genetics and Cell Biology, UIm University, D-89081 Ulm, Germany
}

\section{Biomed Central}

(c) 2013 Renz et al.; licensee BioMed Central Ltd. This is an Open Access article distributed under the terms of the Creative Commons Attribution License (http://creativecommons.org/licenses/by/2.0), which permits unrestricted use, distribution, and reproduction in any medium, provided the original work is properly cited. 


\section{Background}

The classical Hartwell screen revealed, besides many other genes involved in cell cycle regulation of yeast, mutations in the four genes $C D C 3, C D C 10, C D C 11$ and $C D C 12$. These were later classified as members of the septins, a new family of proteins in the budding yeast Saccharomyces cerevisiae [1]. Another mitotic septin, Shs1, was identified about a decade later [2]. The four essential septins $\mathrm{Cdc} 3, \mathrm{Cdc10}, \mathrm{Cdc11}$ and $\mathrm{Cdc12}$ were subsequently shown to be localized below the plasma membrane at the bud neck, where they initially form a ring at the cortical site of the future bud. This ring expands into an hour-glass shaped collar that splits at the onset of cytokinesis in two rings with the contractile actin ring positioned between them [3]. The septin collar is supposed to define the diffusion barrier between the mother cell and the growing bud [4]. Septins also act as a scaffold for various other proteins involved in cell wall deposition, cell cycle control and cytokinesis [5,6].

All septins are GTPases that share structural similarities with the small GTPases of the Ras family. The central GTPase domain containing conserved nucleotide binding regions as well as switch $\mathrm{I}$ and switch II stretches is extended at its $\mathrm{N}$ - and C-terminus by further septin-specific structural elements $[7,8]$. S. cerevisiae septins have been expressed and purified from E.coli. The purified four essential septins assemble into a linear heterooctameric rod that displays the following order: Cdc11-Cdc12-Cdc3-Cdc10-Cdc10-Cdc3-Cdc12Cdc11. These septin rods can be induced to form long septin filaments in vitro by lowering the salt concentration of a buffer that otherwise keeps the rods stable in solution [9].

From the crystal structure of the human heterotrimeric SEPT2-SEPT6-SEPT7 complex [10], two different contact sites between alternate septin subunits had been deduced: A G-interface build by two G-domains and a NC-interface involving the $\mathrm{N}$ - and $\mathrm{C}$-terminal residues of two neighboring septins.

Septins were shown to associate with cellular membranes, actin filaments and microtubules [11,12]. In mammalian cells, septins are required for correct mitotic spindle organization, chromosome segregation, spindle elongation and midbody abscission [13-15]. A potential role in cancerogenesis is under debate [16].

Different approaches are currently used to label a protein with a sensor to monitor certain aspects of its behavior in vitro or in vivo. Among those, the SNAP tag represents a very flexible tool to covalently attach different "functionalities" such as fluorescence, affinity or reactive groups to any protein of interest $[17,18]$. The SNAP-tag technology is based on an engineered version of the DNA repair protein $\mathrm{O}^{6}$-alkylguanine-DNAalkyltransferase [19] which transfers alkyl adducts from the $\mathrm{O}^{6}$-position of guanine covalently to its reactive cysteine independent of the nature of the alkyl group. The method has already been widely used for various in vivo and in vitro applications [20-22]. Particularly, labeling of SNAP tag fused septins with fluorescent Benzylguanine derivatives in living yeast cells already demonstrated that the tag and its label does not interfere with the functions of the septin subunit and its incorporation into rods and filaments [20].

In order to facilitate biochemical studies of the septins in vitro, we genetically fused a SNAP tag to one subunit of the yeast septin rod. The E.coli expressed SNAP tagged septin rods allowed us to (i) label them with fluorescent compounds to visualize filament formation by microscopy in vitro and to (ii) immobilize septin rods via an attached biotin moiety to a surface plasmon resonance (SPR) sensor chip to estimate their stability and to detect and quantify physical interactions between septin rods and their binding partners independently of posttranslational modifications.

\section{Results and discussion}

Purification of SNAP tag modified septin rods from E.coli

Before establishing a protocol for the SNAP tagged septin complexes, we first aimed at optimizing the published protocol for the purification of septin rods [9]. We co-expressed the four different septin subunits each fused to a short peptide tag to aid further purification and/or downstream assays: Cdc12 carried the 6-his tag for purification, Cdc10 and Cdc11 carried a S tag and Cdc3 was fused to a N-terminal FLAG tag. Expression was carried out at low temperature over night. As we noticed that recombinant septin rods tended to be unstable in the crude E.coli extract, we shortened the purification procedure to less than 6 hours. Specifically, applying a gradient step elution procedure in the IMAC chromatography removed already major contaminants and allowed us to circumvent the time-consuming gel filtration step. The modified protocol yielded around 2.0 mg of intact septin rods per liter expression culture with a purity of $>85 \%$ (judged by SDS-PAGE; Figure $1 \mathrm{~A}$ ).

We subsequently applied our established purification protocol to SNAP tag modified septin rods. Here, the SNAP tag replaced the $\mathrm{S}$ tag of the Cdc10 subunit.

The SNAP tag labeled rods behaved like their S tag labelled counterparts during expression and purification from E.coli extracts. To investigate whether the SNAP tag altered the stability and composition of the purified septin rods, we compared the isoelectric points and the aggregation states of the two different samples of purified septin rods (Figure 1B). Both SNAP tagged and untagged rods could be detected as a single band in an IEF gel after Coomassie staining. The estimated pI of 6.0 is in good agreement with the in silico predicted pI of 


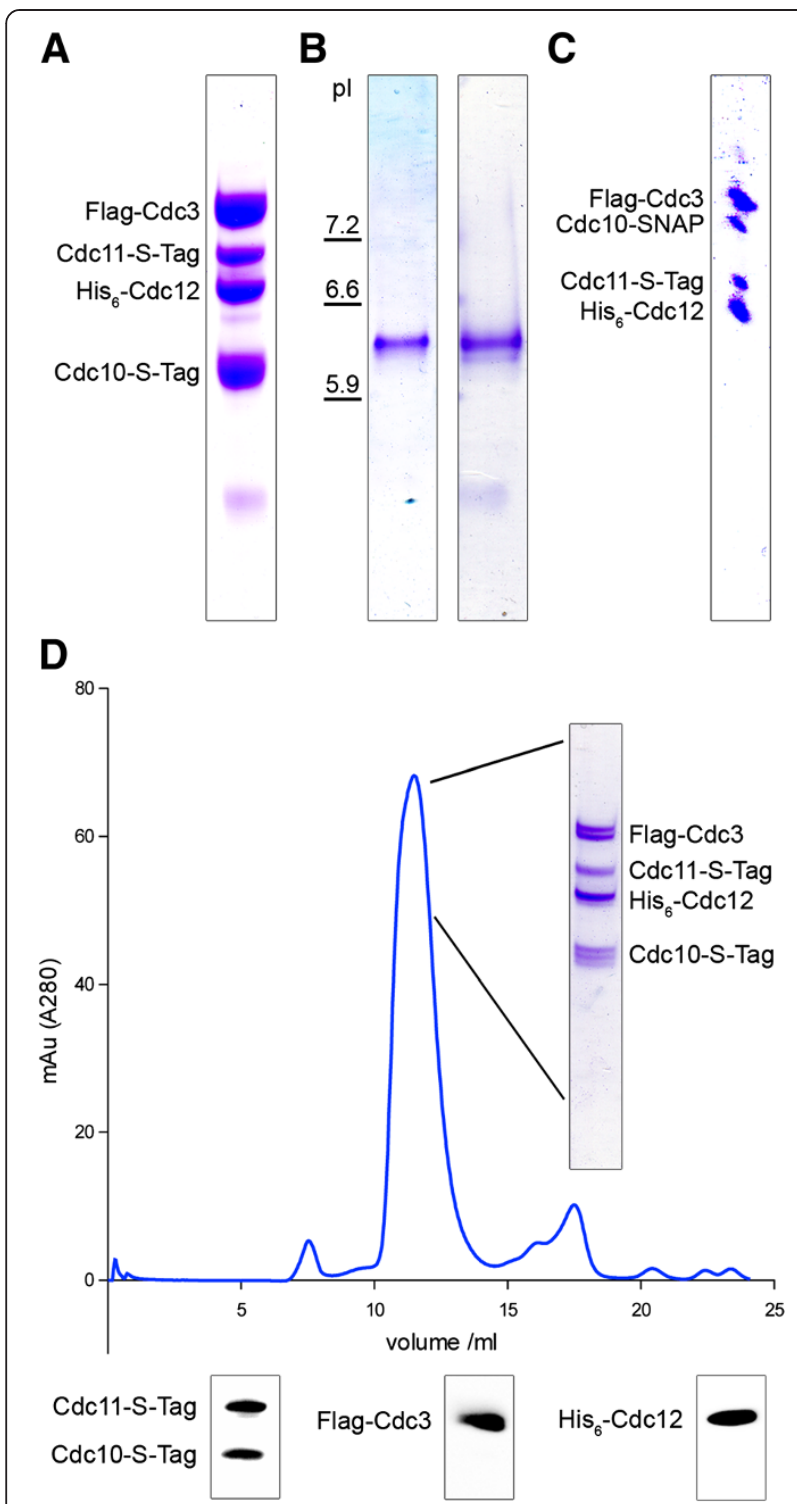

Figure 1 Purification of recombinant septin rods from E. coli. A. Coomassie stained SDS-PAGE of the purified septin rod. B. Isoelectric focussing of purified septin rods (left panel) or SNAP tagged septin rods (right panel). C. PAGE separation of the excised band from the IEF for SNAP tagged septin rods. All four septins are present in the complex. D. Analytical size exclusion chromatography of purified septin rods. The rods elute in one prominent peak containing all four constituents. The single septins can be detected by Western blot using antibodies against Cdc3 and the attached 6his tag and S tag, respectively.

5.8. As the calculated pIs of the isolated septin subunits vary between 4.9 (Cdc11) and 7.8 (Cdc12), a loss of a subunit should have resulted in a readily detectable change in mobility during the IEF analysis. The single band corresponding to the septins was excised from the IEF gel and subjected to denaturing SDS-PAGE. Four bands, corresponding to the four septins, could be detected after Coomassie staining in both septin-rod samples (shown in Figure 1C for SNAP tagged rods). We further investigated the integrity of the purified samples by analytical size exclusion chromatography. Both SNAP tagged and untagged rods eluted in a single peak from the column. The near stoichiometric presence of all four septins in the peak fraction was confirmed by SDS-PAGE and Western blot analysis (Figure 1D).

\section{Formation of septin filaments in vitro}

Septin filament formation can be induced in vitro by lowering the salt of the buffer below $100 \mathrm{mM}$ [9]. A solution containing $2 \mu \mathrm{M}$ SNAP tagged septin rods in high-salt buffer was labeled with an excess of BG-TMR and subsequently dialyzed into low salt buffer and incubated at $4^{\circ} \mathrm{C}$ over night. The labeled filaments were then pelleted and captured on poly-L-Lysine coated glass slides for observation under a fluorescence microscope. We observed filaments with lengths ranging from 1.4 to $14.5 \mu \mathrm{m}$ (Figure 2). Filament formation was strictly suppressed by high salt. To evaluate whether the fusion to the SNAP tag interferes with essential features of the formed filaments, we induced filament formation by dialysis in low salt buffer and added $2 \mu \mathrm{M}$ purified Bni5SNAP fusion to the mixture. Bni5 is an already described septin-interacting protein that colocalizes with septin higher-order structures in yeast [23]. The reported interaction data however fell short of demonstrating that Bni5 physically binds to septin filaments. After $60 \mathrm{~min}$ of septin/Bni5-SNAP co-incubation, the samples were pelleted and analyzed by SDS-PAGE and Western blot. The appearance of Bni5-SNAP in the pellet fraction was strictly depending on the presence of the septins and only observed under filament-forming conditions thus proving that Bni5 also binds to intact septin filaments (Figure 3A).

\section{SPR with SNAP tag modified septin rods}

The dynamic properties of protein-protein interactions influence the behavior of protein complexes or larger protein interaction networks, and are instrumental in understanding the action of drugs on a certain interaction [24,25]. However, most quantitative data on protein interactions are derived under equilibrium conditions (e.g. FRET or calorimetry). In contrast, chip based biosensors in combination with SPR allow to directly measure the on- and off-rates of proteinprotein interactions in vitro [26].

The gathered evidence of the native-like properties of the SNAP tagged septin rods encouraged us to couple an affinity handle to the SNAP tag for immobilization of the entire rod to a SPR chip for performing quantitative binding experiments. We labeled SNAP tagged septin rods with BG-biotin in solution and used an anti-biotin antibody as capturing molecule on the surface of the 

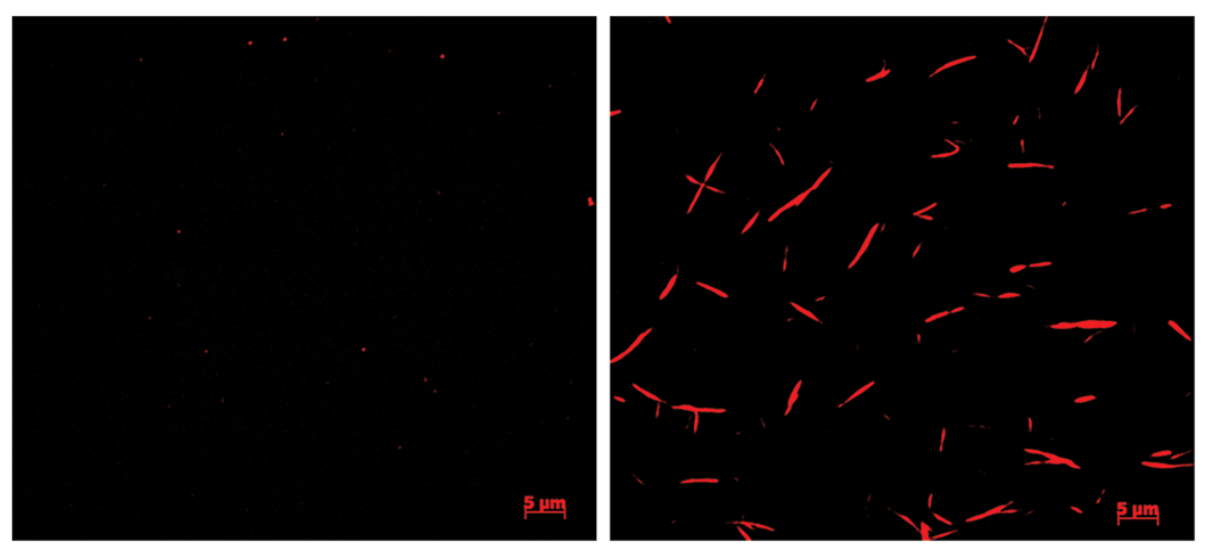

Figure 2 Fluorescence microscopy of SNAP tagged, TMR labeled septin filaments. Left panel: No filaments are formed under high salt conditions. Right panel: Filaments in low salt conditions.

chip. This procedure allowed us to mildly regenerate the chip surface by removing the biotinylated SNAP tag fusion protein and thus enabled us to use one and the same chip for different SNAP tagged septin rod preparations. This is an advantage over published methods that employ chips covalently coupled with $\mathrm{BG}-\mathrm{NH}_{2}$ substrates. Here SNAP tagged fusions are directly captured from solution, thus avoiding previous labeling [27]. However, regeneration of the surface (i.e. removing the analyte when performing kinetic experiments with increasing analyte concentrations) has to be determined individually for each ligand.

The septin rod was coupled through its central Cdc10 subunit to the surface leaving the more peripheral subunits free to dissociate from the rod. This assay configuration was used to determine the stability of the entire

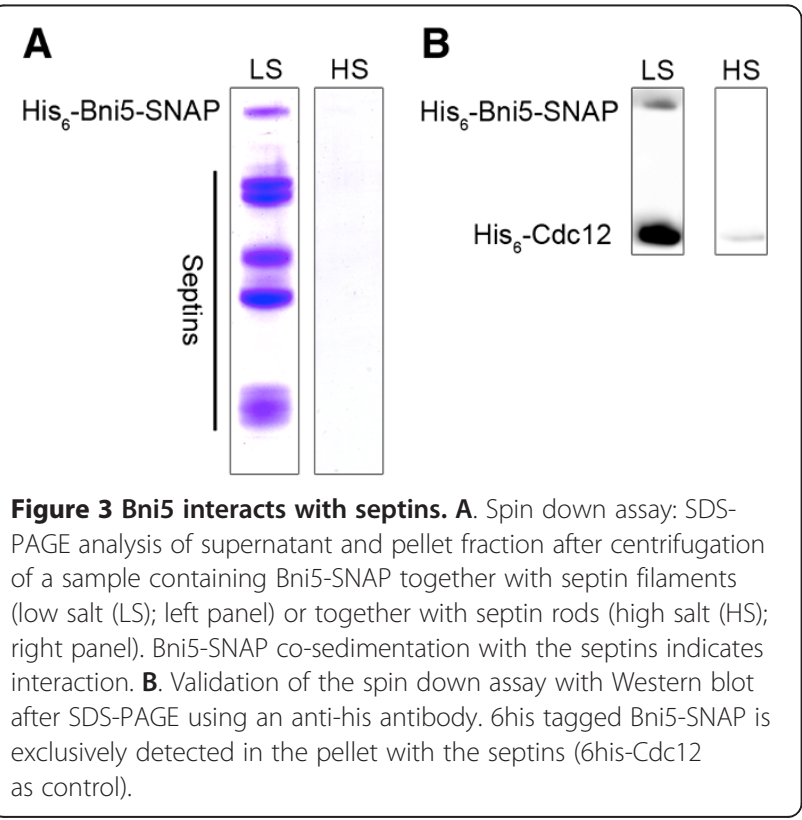

rod. We captured septin rods via the SNAP tag and followed the potential dissociation of its subunits by measuring the decline in response units. The constant response units over hours of washing might either indicate a high kinetic stability of the rod or the dissociation of the peripheral subunits already during the capture procedure (data not shown). The N-terminally fused $\mathrm{S}$ tag to the Cdc11 subunit of the rod allowed us to control the integrity of the captured rod preparation by injecting the antibody against the $\mathrm{S}$ tag in saturating concentrations. During the washing of the chip surface for two hours, the baseline dropped approximately to the capture level very probably indicating the dissociation of the antibody from the $\mathrm{S}$ tag. Dissociation of intact rods from the sensor chip surface is unlikely, as the surface is coated to saturation with the anti-biotin antibody.

Subsequently, another pulse of anti S tag antibody was injected. The resulting response unit was almost as high as the one obtained after the first injection, indicating the same amount of binding of the antibody to the peripheral Cdc11 subunit and thus the complete integrity of the rod during the whole experiment (Figure 4A).

Using the SPR sensor chip-immobilized septin rods, we next analyzed the interaction between Bni5 and the septins. By plotting the analyte response against the respective analyte concentration, we calculated a dissociation constant of $3.13 \times 10^{-7} \mathrm{M}\left( \pm 1.93 \times 10^{-7}, \mathrm{~N}=4\right)$ for the interaction between Bni5 and septin rods (Figure 4B). A very similar dissociation constant of $2,53 \times 10^{-7} \mathrm{M}$ $\left( \pm 1.37 \times 10^{-7}, \mathrm{~N}=3\right)$ was obtained when we fitted the analyte response sensograms of the experiments with a suitable model (Figure 4C). Here, a two state reaction model was used to compensate for background binding of Bni5 to the antibody-coated chip surface. The on- and offrates of the interaction were also calculated from a twostate reaction fit to the respective analyte response 


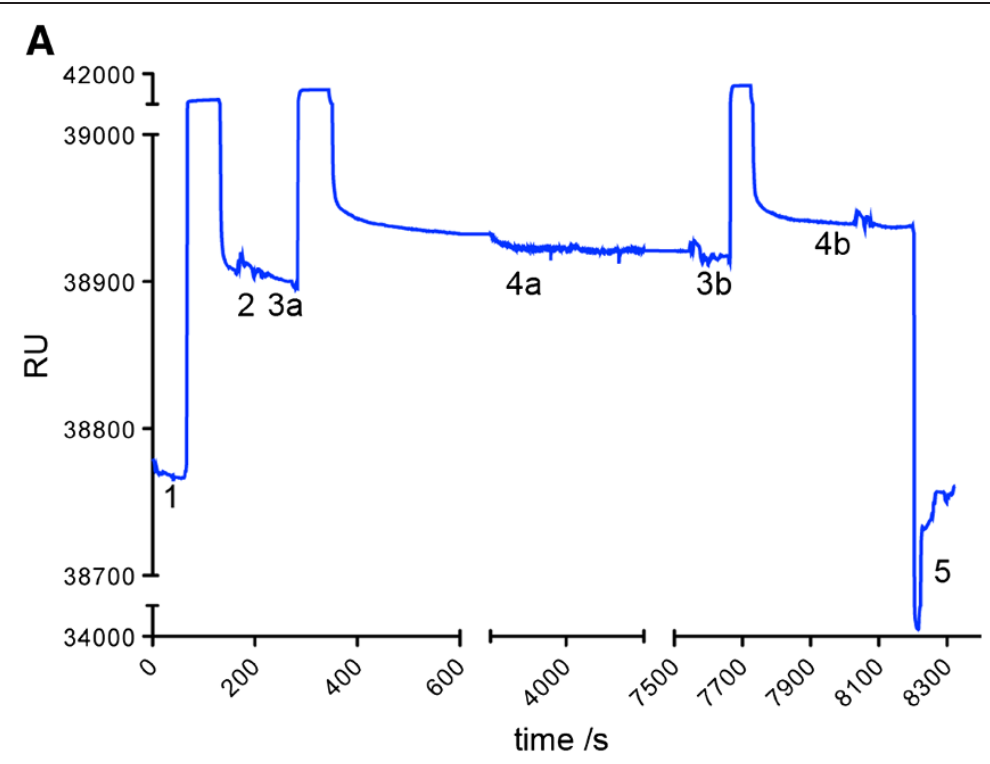

B
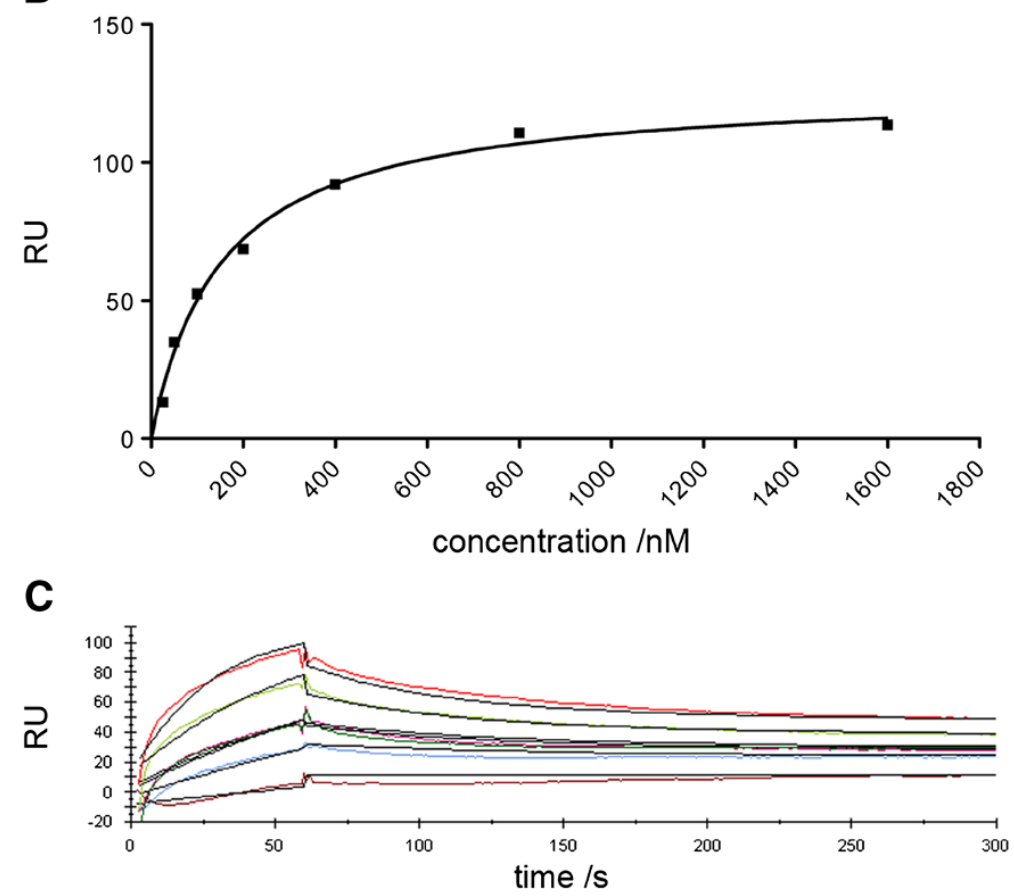

Figure 4 Analysis of septin rod stability and the interaction of Bni5 with the rod via SPR. A. Septin rods are stable when immobilized to SPR sensor chips. (1) Capture of biotinylated rods to the anti-biotin antibody coated SPR chip, (2) capture baseline, (3a,b) injection of anti S tag antibody, $(4 a, b)$ dissociation, (5) regeneration of the chip surface. B. Determination of the $K_{D}\left(3.13 \times 10^{-7} \mathrm{M}\right)$ of the interaction of Bni5 with immobilized septin rods from the fit of the analyte response plotted against the respective analyte concentration (representative plot from a series of experiments). $C$. Determination of the kinetics parameters from the same dataset as shown in $\mathrm{B}$. The analyte response sensograms were fitted with a two state reaction model to obtain $\mathrm{K}_{\mathrm{D}}\left(2,53 \times 10^{-7} \mathrm{M}\right), \mathrm{k}_{\text {on }}\left(5,1 \times 10^{4} \mathrm{M}^{-1} \mathrm{~s}^{-1}\right)$ and $\mathrm{k}_{\text {off }}\left(0,037 \mathrm{~s}^{-1}\right)$ (representative plot from a series of experiments).

sensograms $\left(\mathrm{k}_{\text {off }}=0,037 \mathrm{~s}^{-1} \pm 0,019, \mathrm{~N}=4 ; \mathrm{k}_{\text {on }}=5,1 \times 10^{4}\right.$ $\left.\mathrm{M}^{-1} \mathrm{~s}^{-1} \pm 0,45 \times 10^{4}, \mathrm{~N}=3\right)$.

To our knowledge these experiments provide the first quantitative description of an interaction between functional septin rods and one of its ligands. Our measurements provide no indication that either phosphorylation of Bni5 [28] or the posttranslational modifications of the septins are a prerequisite for a successful interaction: Bni5 and septins both purified from E.coli should lack all posttranslational modifications that are 
reported to occur in yeast $[29,30]$. Whether phosphorylation of Bni5 affects the affinity or the kinetics if its interaction with the septins remains to be explored.

\section{Conclusions}

We provide methods for purifying SNAP tag labeled septin rods in high yield and purity. Filament formation and binding assays confirmed that the labeled septin rods display native-like properties. The advantage of the approach was documented by labeling the SNAP tagged septin rods with two different BG-derivatives. Coupling a fluorescent dye allowed to monitor septin filament formation whereas coupling of Biotin allowed immobilizing septin rods on SPR sensor chips. The septin immobilized SPR sensor chips were used to determine for the first time the stability of septin rods and to exemplarily characterize the kinetic and equilibrium constants of an interaction between septin rods and its ligand Bni5.

In contrast to approaches using an individual tag for each application the SNAP tag of one septin preparation can be used for various analytical downstream applications.

The herein introduced methods thus significantly broaden the spectrum of analytical tools to investigate this important but still poorly characterized new class of cytoskeletal structures.

\section{Methods}

\section{Cloning, expression and purification of septin rods}

We aimed at purifying the essential septins Cdc12, Cdc11, Cdc3 and Cdc10 simultaneously from the same cell by cloning each two ORFs (Cdc12/Cdc11 and Cdc3/ $\mathrm{Cdc} 10)$ in one bicistronic expression vector. The four essential septins CDC3, CDC10, CDC11 and CDC12 were amplified by PCR from genomic DNA and cloned in two bicistronic vectors with compatible replicons (pACYCDuet1 and pET-Duet1, Novagen). A list of all PCR primers used in this work is provided in the Additional file 1.

FLAG-CDC3 (with DYKD coding for the FLAG Tag) and $\mathrm{CDC10}$ fused to the $\mathrm{S}$ tag or to an engineered version of the SNAP tag [19] were cloned in pACYCDuet1 leading to pACYC-Duet1-FLAG-CDC3-CDC10-S or pACYC-Duet1-FLAG-CDC3-CDC10-SNAP respectively. The 6his tag of the first multiple cloning site was removed. $\mathrm{CDC} 12$ in frame with the 6his tag and CDC11 fused to the $S$ tag were cloned in pETDuet1 leading to pET-Duet1-6his-Cdc12-CDC11S. The plasmids pACYC-Duet1-FLAG-CDC3-CDC10-S or pACYC-Duet1-FLAG-CDC3-CDC10-SNAP and pETDuet1-6his-CDC12-CDC11-S were co-transformed in the $E$. coli strain BL21(DE3). The transformed cells were grown in Super Broth (SB)-Medium (containing Ampicillin and Chloramphenicol) to an $\mathrm{OD}_{600 \mathrm{~nm}}$ of 1.0, induced with $1.0 \mathrm{mM}$ isopropyl- $\beta$-D-thiogalactoside for $21 \mathrm{~h}$ at $18^{\circ} \mathrm{C}$, harvested by centrifugation, washed once with PBS and stored at $-80^{\circ} \mathrm{C}$. Frozen cells were resuspended in extraction buffer $(300 \mathrm{mM} \mathrm{NaCl}, 2 \mathrm{mM} \mathrm{MgCl} 2,15 \mathrm{mM}$ imidazole, $5 \mathrm{mM} \beta$-mercatoethanol, $40 \mu \mathrm{M}$ GDP, 12\% (v/ v) glycerol, $0.1 \%(\mathrm{v} / \mathrm{v})$ Tween20, Complete Protease Inhibitors Cocktail (Roche), $50 \mathrm{mM}$ Tris- $\mathrm{HCl} \mathrm{pH} \mathrm{8.0)} \mathrm{and}$ cell lysis was performed by addition of $1.0 \mathrm{mg} / \mathrm{ml}$ lysozyme (30 min on ice) and sonication (Branson Sonifier A250). The resulting lysate was clarified by centrifugation at $40,000 \mathrm{~g}$ for $10 \mathrm{~min}$ at $4{ }^{\circ} \mathrm{C}$ and the supernatant was loaded on an ÄktaPurifier Chromatography System (GE Healthcare). The first step of purification was an immobilized metal affinity chromatography (IMAC) by capturing the septin rod via the 6his tag at the $\mathrm{N}$ terminus of Cdc12 on a HisTrap-HP column with $5 \mathrm{ml}$ bed volume (GE Healthcare) equilibrated with IMAC buffer A $(300 \mathrm{mM} \mathrm{NaCl}, 2 \mathrm{mM} \mathrm{MgCl} 2,15 \mathrm{mM}$ Imidazole, $5 \mathrm{mM}$ Dithiothreitol (DTT), 12\% (v/v) glycerol, 50 $\mathrm{mM}$ Tris- $\mathrm{HCl} \mathrm{pH} \mathrm{8.0).} \mathrm{The} \mathrm{proteins} \mathrm{were} \mathrm{eluted} \mathrm{from}$ the column by applying consecutive Imidazole segment gradients with $3 \%, 25 \%$ and $100 \%$ IMAC buffer B (500 $\mathrm{mM} \mathrm{NaCl}, 2 \mathrm{mM} \mathrm{MgCl} 2,500 \mathrm{mM}$ Imidazole, $5 \mathrm{mM}$ DTT, $12 \%(\mathrm{v} / \mathrm{v})$ glycerol, $50 \mathrm{mM}$ Tris- $\mathrm{HCl} \mathrm{pH} \mathrm{8.0).} \mathrm{The}$ septin rods eluted at $25 \%$ IMAC B. Septin rod-containing fractions were pooled and buffered in BisTris $\mathrm{pH} 7.5$ (prepared by the Buffer Prep device of the chromatography system) using PD10 desalting columns (GE Healthcare).

The second purification step was performed via a Resource Q anion exchange column (GE Healthcare) in Buffer Prep mode (BisTris pH 7.0) and elution with a linear $\mathrm{NaCl}$ gradient. The septin rod eluted at $600 \mathrm{mM}$ $\mathrm{NaCl}$.

The septin-containing fractions were pooled, buffered in Tris-buffer (50 mM Tris- $\mathrm{HCl} \mathrm{pH} \mathrm{8.0,300} \mathrm{mM} \mathrm{NaCl)}$ containing $10 \%(\mathrm{v} / \mathrm{v})$ glycerol using PD10 desalting columns and concentrated using Amicon Ultra Centrifugal Units (Millipore). Protein concentration was determined at $280 \mathrm{~nm}$ in a NanoDrop ND-1000 spectral photometer (Peqlab) with calculated extinction coefficients (Septin rod: $251.2 \mathrm{mM}^{-1} \mathrm{~cm}^{-1}$, Septin rod-SNAP: $293.5 \mathrm{mM}^{-1}$ $\mathrm{cm}^{-1}$ ) and molecular weights (Septin rod: $397.7 \mathrm{kDa}$, Septin rod-SNAP: $432.5 \mathrm{kDa}$ ) or with Bradford [31]. For predictions of the molecular weight and extinctions coefficients the bioinformatic tools provided on the EXPASY server (www.expasy.org) were used. Purified complexes were aliquoted and stored at $-20^{\circ} \mathrm{C}$.

The activity of the SNAP tag was confirmed by labeling the Cdc10-SNAP with BG-TMR (New England Biolabs). The SNAP tagged septin rod was incubated with an excess of substrate for $30 \mathrm{~min}$ at RT, separated by SDS-PAGE and TMR fluorescence in the gel was finally visualized through the Cy3-Filter of a Stella Documentation System (Raytest) (data not shown). 
Isoelectric focusing (IEF) was performed using precast pH 3-10 IEF gels (Invitrogen) according to the manufacturer's instructions.

For analytical size exclusion chromatography, purified septin rods were loaded on a Superose 6 10/300 GL column (GE Healthcare) pre-equilibrated with $300 \mathrm{mM}$ $\mathrm{NaCl}, 50$ mM Tris-HCl pH 8.0.

\section{Expression and purification of Bni5 and Bni5-SNAP}

BNI5 from S.cerevisiae was PCR amplified from genomic DNA, cloned in a pET15b derived expression plasmid [32] with or without previously inserted SNAP tag and expressed as 6his tagged protein in E.coli strain BL21 (DE3) in SB medium at $18^{\circ} \mathrm{C}$. Purification was done by IMAC, followed by size exclusion chromatography against HBSEP buffer (10 mM HEPES, $150 \mathrm{mM} \mathrm{NaCl}, 3$ mM EDTA, 0.05\% Tween 20, pH 7.4) using a Superdex 200 16/60 column (GE Healthcare).

\section{Analysis of filament formation}

Analysis of septin filament formation was performed using an established protocol [33] with some modifications: The purified septin rods were buffered in high salt buffer (300 mM NaCl, 50-200 mM Tris- $\mathrm{HCl}$ pH 8.0) using NAP5 columns (GE Healthcare), diluted to $2 \mu \mathrm{M}$ and clarified by centrifugation at $100,000 \mathrm{~g}$ for $1 \mathrm{~h}$ at $4^{\circ} \mathrm{C}$. The supernatant was subsequently dialyzed (MFMembrane Filters $0.025 \mu \mathrm{m}$; Millipore) into low salt buffer (50 mM NaCl, 50-200 mM Tris-HCl pH 8.0) for $1 \mathrm{~h}$ on ice and incubated for $16 \mathrm{~h}$ at $4^{\circ} \mathrm{C}$.

Septin filaments were subsequently pelleted at 100,000 $\mathrm{g}$ for $1 \mathrm{~h}$ at $4^{\circ} \mathrm{C}$. Filament formation was analyzed by applying the supernatant and the pellet on a SDS-PAGE followed by coomassie staining. BG-TMR labeling of SNAP tagged septin rods was performed in high salt conditions prior to the first centrifugation step by adjusting the septin mixture to $8 \mu \mathrm{M}$ BG-TMR. Filaments were transferred to poly-L-lysine-coated slides (Science Services), fixed with 3\% (w/v) paraformaldehyde for $15 \mathrm{~min}$ at RT, blocked with $10 \%(\mathrm{w} / \mathrm{v})$ bovine serum albumin (30 min at RT), washed with PBS and embedded with VectaShield Hard Set mounting medium (Vector Laboratories). TMR labeled septin filaments were subsequently visualized by fluorescence microscopy using an Axio-Observer Z1 SD confocal microscope (Zeiss). The length of the filaments was determined using the evaluation tool implemented in the aquisition software AxioVision (version 4.8.1, Zeiss).

For the qualitative monitoring of septin filament interaction, $2 \mu \mathrm{M}$ Bni5-SNAP was added to the reaction after filament formation. The mixture was incubated for $1 \mathrm{~h}$ prior to centrifugation ("spin down assay").

\section{Analysis of septin-interacting proteins by SPR}

Kinetics of septin-interacting proteins were measured by surface plasmon resonance using a Biacore X100 system (GE Healthcare).

For the capture of biotinylated septin rods, the surface of a CM5 Chip (GE Healthcare) was previously coated with an anti-Biotin-antibody (US Biologicals) as capture molecule using NHS ester chemistry in $10 \mathrm{mM}$ Sodium actetate pH 5.0 with HBSEP as running buffer.

Purified SNAP tagged septin rods (ligand protein) were covalently labeled with BG-Biotin (New England Biolabs) by SNAP tag chemistry in MSP buffer (10 mM MES, $150 \mathrm{mM} \mathrm{NaCl}, 0.05 \%$ Tween 20, pH 7.4) adjusted to $300 \mathrm{mM} \mathrm{NaCl}$. Excess substrate was removed using a NAP5 desalting column.

MSP was used as running buffer for all subsequent experiments. Capture levels for the ligand were in the range of 100 RU.

For determination of the rod stability, an anti-S tag antibody (Novagen; $20 \mu \mathrm{g} / \mathrm{ml}$ in MSP buffer) was injected via a $60 \mathrm{~s}$ pulse after ligand capture and the chip surface system was washed for $2 \mathrm{~h}$ with running buffer before another $60 \mathrm{~s}$ pulse of antibody was injected.

The anti-S tag antibody did not show significant binding to the sensor chip surface coated with anti-biotin antibody (Additional file 2: Figure B).

For the determination of kinetic parameters, purified Bni5 (analyte protein) was prepared in suitable concentrations $(0-1600 \mathrm{nM})$ in MSP buffer. The contact time with the ligand was set to 120 or $180 \mathrm{~s}$ followed by a $600 \mathrm{~s}$ dissociation period for each cycle. SPR detection was performed simultaneously on a reference cell (with capture molecule) and a detection cell with capture molecule and ligand. Background correction (i.e. substraction of the reference cell signal) is performed by default.

Regeneration of the sensor chip between each analyte cycle was performed with a $15 \mathrm{~s}$ injection pulse of $12 \mathrm{mM}$ $\mathrm{NaOH}$. Kinetic constants were calculated with the Biacore Evaluation Software (Version 1.1; GE Healthcare).

\section{Additional files}

Additional file 1: List of PCR primers used in this study.

Additional file 2: SPR control experiments. A. SNAP tagged, unlabeled septin rods are injected on a sensor chip without previous ligand capture. No significant background binding to the capturing molecule can be detected. B. Anti-S tag antibody is injected on a sensor chip without previous ligand capture. No significant background binding to the capturing molecule can be detected.

\section{Abbreviations}

BG: Benzylguanine; IMAC: Immobilized Metal Affinity Chromatography; TMR: Tetramethylrhodamine; MW: Molecular weight (kDa); SPR: Surface plasmon resonance. 


\section{Competing interests}

The authors declare that they have no competing interests.

\section{Authors' contributions}

CR performed the experiments and prepared the figures. TG performed SPR measurements and wrote with contributions of $N J$ and $C R$ the manuscript. $\mathrm{NJ}$ oversaw the project and helped to analyze the data. TG, NJ and CR conceived of the study. All authors read and approved the final manuscript.

\section{Acknowledgements}

We would like to thank Clemens Schneider for technical support. $C R$ is supported by a grant of the Bundesministerium für Bildung und Forschung (BMBF Initiative SysTec 0315690B) to N.J.

Received: 14 March 2013 Accepted: 25 July 2013

Published: 26 July 2013

\section{References}

1. Pringle JR: Origins and development of the septin field. In The Septins. Edited by Hall PA, Russel SEH, Pringle JR. Chichester, UK: John Wiley \& Sons, Ltd; 2008:7-34.

2. Mino A, Tanaka K, Kamei T, Umikawa M, Fujiwara T, Takai Y: Shs1p: A nove member of septin that interacts with spa2p, involved in polarized growth in Saccharomyces cerevisiae. Biochem Biophys Res Commun 1998, 251:732-736.

3. McMurray MA, Thorner J: Septins: molecular partitioning and the generation of cellular asymmetry. Cell Div 2009, 4:18.

4. Dobbelaere J, Barral Y: Spatial coordination of cytokinetic events by compartmentalization of the cell cortex. Science 2004, 305(5682):393-396

5. Gladfelter AS, Zyla TR, Lew DJ: Genetic interactions among regulators of septin organization. Eukaryot Cell 2004, 3(4):847-854.

6. Drees BL, Sundin B, Brazeau E, Caviston JP, Chen GC, Guo W, Kozminski KG, Lau MW, Moskow JJ, Tong A, et al: A protein interaction map for cell polarity development. J Cell Biol 2001, 154(3):549-571.

7. Casamayor A, Snyder M: Molecular dissection of a yeast septin: distinct domains are required for septin interaction, localization, and function. Mol Cell Biol 2003, 23(8):2762-2777.

8. Trimble WS: Septins: a highly conserved family of membrane-associated GTPases with functions in cell division and beyond. J Membr Biol 1999, 169(2):75-81.

9. Bertin A, McMurray MA, Grob P, Park SS, Garcia G 3rd, Patanwala I, Ng HL, Alber T, Thorner J, Nogales E: Saccharomyces cerevisiae septins: supramolecular organization of heterooligomers and the mechanism of filament assembly. Proc Natl Acad Sci USA 2008, 105(24):8274-8279.

10. Sirajuddin M, Farkasovsky M, Hauer F, Kuhlmann D, Macara IG, Weyand M, Stark $\mathrm{H}$, Wittinghofer A: Structural insight into filament formation by mammalian septins. Nature 2007, 449(7160):311-315.

11. Kozubowski $L$, Larson JR, Tatchell $K$ : Role of the septin ring in the asymmetric localization of proteins at the mother-bud neck in Saccharomyces cerevisiae. Mol Biol Cell 2005, 16(8):3455-3466.

12. Bowen JR, Hwang D, Bai X, Roy D, Spiliotis ET: Septin GTPases spatially guide microtubule organization and plus end dynamics in polarizing epithelia. J Cell Biol 2011, 194(2):187-197.

13. Nakahira M, Macedo JN, Seraphim TV, Cavalcante N, Souza TA, Damalio JC, Reyes LF, Assmann EM, Alborghetti MR, Garratt RC, et al: A draft of the human septin interactome. PLoS One 2010, 5(11):e13799.

14. Spiliotis ET, Kinoshita M, Nelson WJ: A mitotic septin scaffold required for Mammalian chromosome congression and segregation. Science 2005, 307(5716):1781-1785.

15. Estey MP, Di Ciano-Oliveira C, Froese CD, Bejide MT, Trimble WS: Distinct roles of septins in cytokinesis: SEPT9 mediates midbody abscission. J Cell Biol 2010, 191(4):741-749.

16. Russell SE, Hall PA: Do septins have a role in cancer? Br J Cancer 2005, 93(5):499-503.

17. Keppler A, Gendreizig S, Gronemeyer T, Pick H, Vogel H, Johnsson K: A general method for the covalent labeling of fusion proteins with small molecules in vivo. Nat Biotechnol 2003, 21(1):86-89.

18. Gronemeyer T, Godin G, Johnsson K: Adding value to fusion proteins through covalent labeling. Curr Opin Biotechnol 2005, 16(4):453-458.
19. Gronemeyer T, Chidley C, Juillerat A, Heinis C, Johnsson K: Directed evolution of O6-alkylguanine-DNA alkyltransferase for applications in protein labeling. Protein Eng Des Sel 2006, 19(7):309-316.

20. McMurray MA, Thorner J: Septin stability and recycling during dynamic structural transitions in cell division and development. Curr Bio/ 2008, 18(16):1203-1208.

21. Uhlenheuer DA, Wasserberg D, Haase C, Nguyen HD, Schenkel JH, Huskens J, Ravoo BJ, Jonkheijm P, Brunsveld L: Directed supramolecular surface assembly of SNAP-tag fusion proteins. Chemistry 2012, 18(22):6788-6794.

22. Keppler A, Kindermann M, Gendreizig S, Pick H, Vogel H, Johnsson K: Labeling of fusion proteins of O6-alkylguanine-DNA alkyltransferase with small molecules in vivo and in vitro. Methods 2004, 32(4):437-444.

23. Lee PR, Song S, Ro HS, Park CJ, Lippincott J, Li R, Pringle JR, De Virgilio C, Longtine MS, Lee KS: Bni5p, a septin-interacting protein, is required for normal septin function and cytokinesis in Saccharomyces cerevisiae. Mol Cell Biol 2002, 22(19):6906-6920.

24. Ozbabacan SE, Engin HB, Gursoy A, Keskin O: Transient protein-protein interactions. Protein Eng Des Sel 2011, 24(9):635-648.

25. Phizicky E, Bastiaens Pl, Zhu H, Snyder M, Fields S: Protein analysis on a proteomic scale. Nature 2003, 422(6928):208-215.

26. Rich RL, Cannon MJ, Jenkins J, Pandian P, Sundaram S, Magyar R, Brockman J, Lambert J, Myszka DG: Extracting kinetic rate constants from surface plasmon resonance array systems. Anal Biochem 2008, 373(1):112-120.

27. Kindermann M, George N, Johnsson N, Johnsson K: Covalent and selective immobilization of fusion proteins. J Am Chem Soc 2003, 125(26):7810-7811.

28. Nam SC, Sung H, Kang SH, Joo JY, Lee SJ, Chung YB, Lee CK, Song S: Phosphorylation-dependent septin interaction of Bni5 is important for cytokinesis. J Microbiol 2007, 45(3):227-233.

29. Ho CW, Chen HT, Hwang J: UBC9 autosumoylation negatively regulates sumoylation of septins in Saccharomyces cerevisiae. J Biol Chemsitry 2011, 286(24):21826-21834.

30. Chi A, Huttenhower C, Geer LY, Coon JJ, Syka JEP, Bai DL, Shabanowitz J, Burke DJ, Troyanskaya OG, Hunt DF: Analysis of phosphorylation sites on proteins from Saccharomyces cerevisiae by electron transfer dissociation (ETD) mass spectrometry. Proc Natl Acad Sci USA 2007, 104(7):2193-2198.

31. Bradford MM: A rapid and sensitive method for the quantitation of microgram quantities of protein utilizing the principle of protein-dye binding. Anal Biochem 1976, 72:248-254.

32. Iffland A, Tafelmeyer P, Saudan C, Johnsson K: Directed molecular evolution of cytochrome c peroxidase. Biochemistry 2000, 39(35):10790-10798.

33. Farkasovsky M, Herter P, Voss B, Wittinghofer A: Nucleotide binding and filament assembly of recombinant yeast septin complexes. Biol Chem 2005, 386(7):643-656.

\section{doi:10.1186/1472-6750-13-60}

Cite this article as: Renz et al:: An efficient protocol for the purification and labeling of entire yeast septin rods from E.coli for quantitative in vitro experimentation. BMC Biotechnology 2013 13:60.

\section{Submit your next manuscript to BioMed Central and take full advantage of:}

- Convenient online submission

- Thorough peer review

- No space constraints or color figure charges

- Immediate publication on acceptance

- Inclusion in PubMed, CAS, Scopus and Google Scholar

- Research which is freely available for redistribution 